\title{
The effect of worked material hardness on stone tool wear
}

2 Alice Rodriguez ${ }^{1 *}$, Kaushik Yanamandra ${ }^{2}$, Lukasz Witek ${ }^{3,4}$, Zhong Wang $^{3}$, Rakesh K. Behera ${ }^{2}$, Radu

$3 \quad$ Iovita $^{1,5 *}$

4

$5{ }^{1}$ Anthrotopography Laboratory, Center for the Study of Human Origins, Department of Anthropology, New York University, New York, USA.

$6 \quad{ }^{2}$ Composite Materials and Mechanics Laboratory, Mechanical and Aerospace Engineering Department, New York University, Tandon School of

7 Engineering, Brooklyn, New York, USA.

$8{ }^{3}$ Department of Biomaterials and Biomimetics, New York University College of Dentistry, New York, USA.

$9 \quad{ }^{4}$ Department of Biomedical Engineering, New York University Tandon School of Engineering, New York, USA.

$10{ }^{5}$ Department of Early Prehistory and Quaternary Ecology, Eberhard Karls University of Tübingen, Germany.

11

$12 *$ Corresponding author

13 aar596@nyu.edu (A.R.)

14 iovita@nyu.edu $($ R. I.)

\section{Abstract}

16 The identification of ancient worked materials is one of the fundamental goals of lithic use wear

17 analysis and one of the most important parts of understanding how stone tools were used in the

18 past. Given the documented overlaps in wear patterns generated by different materials, it is

19 imperative to understand how individual materials' mechanical properties might influence wear

20 formation. Because isolating physical parameters and measuring their change is necessary for

21 such an endeavor, controlled (rather than replicative) experiments combined with objective

22 measurements of surface topography are necessary to better grasp how polish formed on stone

23 tools. Therefore, we used a tribometer to wear natural flint surfaces against five materials (bone, 
24 antler, beech wood, spruce wood, and ivory) under the same force, and speed, over one, three,

25 and five hours. The study aimed to test if there is a correlation between polish formation and the

26 hardness of the worked material. We measured each raw material's hardness using nano-

27 indentation test, and we compared the surface texture of the polished flint bits using a 3D optical

28 profilometer. The interfacial detritus powder was analyzed with a scanning electron microscope

29 to look for abraded flint particles. We demonstrate that, contrary to expectation, softer materials,

30 such as wood, create smoother polishes than hard ones, such as ivory.

\section{Introduction}

32 Along with the study of ancient residues, microscopic use wear analysis (MWA) is one of the

33 two major and complementary methods of lithic traceology [1], the science of forensically

34 interpreting ancient stone tool use. Despite its immense interpretive potential, its early

35 spectacular results [e.g., 2] were tempered by the blind testing crisis of the 1980s [3,4], which

36 put into question different researchers' ability to identify the same worked materials. Despite

37 many advances since the 1980s [see 5-7,8 for reviews], the identification of different worked

38 materials is still considered an insufficiently developed area. This is a major setback for

39 prehistorians, because residues are not always preserved, and knowing which materials were

40 worked can make an enormous difference in interpreting area or site function, or in claims about

41 style and cultural transmission, etc.

42 The problem is manifold: not all polishes are equally easy to see and interpret, and not all types

43 of data deliver the same answers. Some types of materials, such as cereals $[9,10]$ or antler, bone,

44 and ivory (ABI) have been identified consistently in blind tests or have been separated

45 quantitatively in experimental settings with varying degrees of success (see [11-15]). Traces left 
by others, especially by soft and elastic materials, such as meat, tendons, etc., are less identifiable [16]. Several quantitative studies did report success in distinguishing target materials, but, even here, within-group similarities were not adequately explained. For example,

49 Stevens et al.'s [15] discriminant functions grouped antler with plant polishes. Moreover, some studies could not clearly distinguish wood [17] and hide polish [13] from unworked areas. In one

51 of the most recent studies, Ibáñez et al. [12: 1188] report a similar overlap between some contact

52 materials in their quantitative analysis as those noted by both other quantitative studies and also

53 in blind tests [e.g., 18], despite studying one of the largest samples to date. Therefore, because

54 the processes that lead to these differences and similarities in polish are not sufficiently well

55 understood, we cannot understand why attempts to classify polishes produced by different

56 materials fail or produce ambiguous results when they do.

57 One obvious way to resolve this problem is to study the mechanical properties of prehistoric

58 target (worked) materials and test their effects on wear development. Unfortunately, these materials are not commonly studied by tribologists, as they have no industrial applications. They 60 are also more variable than industrial ones, as the available data show. For example, the varying amount of mineralization in bone, ivory, and antler causes mechanical properties to also vary 62 among species [19], in some cases significantly so [20]). Further, these materials change their 63 properties when they are wet vs. dry [21], or in very cold weather conditions [particularly ivory, see 22]. While some have claimed that this produces different traces on stone tools, why that

65 might happen remains insufficiently explained. Given that it is now widely believed that abrasion 66 is the main process by which stone tools are polished during use [23-25], it makes sense to test 67 first the effect of hardness. Until now, systematic mechanical tests of archaeologically-relevant target materials have not been incorporated into lithic use-wear research. In particular, hardness 
69 tests were carried out on the stones themselves [26-29], but not on the target materials. For this 70 reason, we decided to carry out a test of the target material hardnesses and to evaluate their role 71 in abrading flint.

72

\section{Method}

\section{Experimental setup}

75 To isolate the effect of material hardness while maintaining reasonable costs and effort, we opted

76 for a tightly controlled protocol [30,31]. Flint bits (Baltic/morainic flint from Denmark with

77 nano-crystalline non-oriented chalcedony texture [25]) were rubbed against dry bone, antler, and

78 wood (beechwood and spruce) using a tribometer at room temperature. Five samples of each pair

79 were produced to obtain a suitable number of samples for statistical study. The experiment was

80 sequential; the flint samples were mounted on the tribometer (Nanovea T-50) (Fig 1) and used

81 sequentially for 1,3 , and 5 hours. The tribometer variables were fixed to a load of $20 \mathrm{~N}$, a speed

82 of 35 revolutions per minute and a straight back and forth motion. 

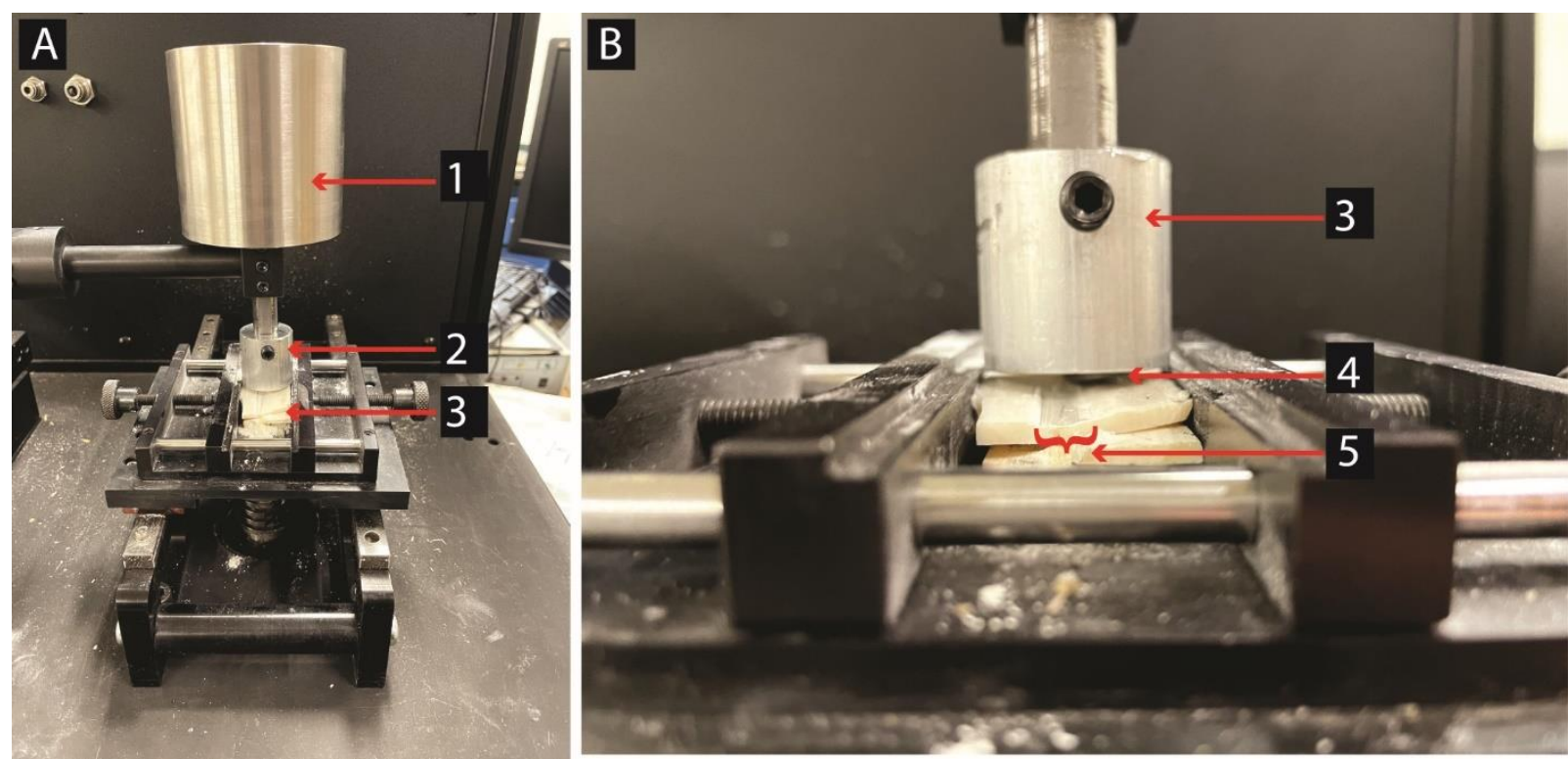

Fig. 1: Tribometer setup. A: overview; 1: Load(20N); 2: sample holder; 3: worked material (ivory). B: close-up; 3: worked

\section{Sample Preparation}

\section{Stone bits}

The stone was first broken in small fragments using a hammer. The stone bits that had a flat natural surface on one side were selected. The opposite side was then adjusted to fit into the tribometer's clamp using a saw (Buehler, ISOMET TM, law speed saw) and then glued (Scotch

91 Create Permanent Super Glue Liquid) to the clamp. The flat natural surface was rubbed against one of the worked materials selected. As polish develops unevenly on the sides of the sharp edge

93 of a tool, this setup helps to create a sufficiently large polished area for carrying out microscopic

94 analyses. The samples were cleaned before every documentation of wear. Because of the

95 metallic nature of the clamp, a harsh cleaning protocol using acid and base was impossible and

96 instead, a mild cleaning process was used. The samples were placed in individual plastic bags 
97 filled with a 10\% neutral soap solution (Valconox, Luminox). They were then immersed into an

98 ultrasonic bath for 15 minutes (Branson $5800,40 \mathrm{kHz}$, at room temperature, $22^{\circ} \mathrm{C}$ ). Then they

99 were rinsed with tap water and put in a distilled water bath for 5 minutes. Finally, samples were

100 left to air-dry.

\section{Hardness test}

102 The hardness of each raw material was measured using a nano-indentation test. The samples

103 were placed in resin using a SamplKwick Fast Cure Acrylic Kit 20-3560 (which contains

104 SamplKwick Powder 20-3562 and SamplKwick Liquid 20-3564). The resin was prepared by a

105 cold molding process done at room temperature by mixing two parts of 20-3562 SamplKwick

106 Powder and one part of 20-3564 SamplKwick and blending thoroughly for 15-20 seconds. Then

107 the mixture was poured into ring molds without delay. Before pouring the mix into the ring

108 molds, we sprayed the cups with Mold release Spray (Buehler Mold Release Spray, 203050008).

109 Finally, the surface of the cast was polished to obtain a smooth surface.

111 The nanoindentation measurements were performed using a nanoindenter (Fig 2) (TI 950,

112 Triboindenter, Hysitron, Minneapolis, MN) equipped with a diamond Berkovitch indenter. Prior

113 to the experiment, the tip area function and the frame stiffness were calibrated using a fused

114 silica standard. Nanoindentation measurements were conducted using the Oliver-Pharr or the

115 quasi-static loading mode [32-34] from which the reduced modulus $\left(E_{r}\right)$ and hardness were

116 estimated (Fig. 3) The initial unloading portion of the load-displacement curve represents purely

117 elastic recovery. The slope of this unloading segment is a measure of the material contact

118 stiffness [35]. The reduced Young's modulus $\left(E_{r}\right)$ can be calculated by 
$119 E_{r}=\frac{1}{2} S \sqrt{\frac{\pi}{A_{\max }}}$

120 where $\mathrm{S}$ is the contact stiffness and $A_{\max }$ is the surface contact area at the maximum depth. The

121 elastic modulus of the indented specimen, $E_{S}$ is computed using

$122 \quad \frac{1}{E_{r}}=\frac{1-v_{S}^{2}}{E_{S}}+\frac{1-v_{i}^{2}}{E_{i}}$

123 where $v_{\mathrm{s}}$ is the Poisson's ratio of the indented specimen and $\mathrm{E}_{i}$ and $v_{i}$ are the Young's modulus

124 and Poisson's ratio, respectively, of the indenter. For a diamond Berkovitch indenter, $E_{\mathrm{i}}$ is equal

125 to $1141 \mathrm{GPa}$ and $v_{i}$ is equal to 0.07 [36]. Since the Poisson's ratio of sedimentary rock ranges

126 between $0.1-0.3$, we have decided to report the $E_{r}$ rather than estimate the $E_{S}$ for modulus

127 comparison (see S1 Appendix).

128

129 In addition, the hardness $(H)$ can be calculated using the maximum load, $P_{\max }$, by

130

$$
H=P_{\max } / A_{c}
$$

131 where $A_{c}$ is the contact area of the indentation. These quasi-static measurements were performed 132 with a $5 \mathrm{~s}$ load time and a $10 \mathrm{~s}$ dwell time at a maximum load of $3000 \mu \mathrm{N}$ for stones and $300 \mu \mathrm{N}$ 133 for, spruce wood, beech wood, bone, ivory, and antler (Table 1). 
A

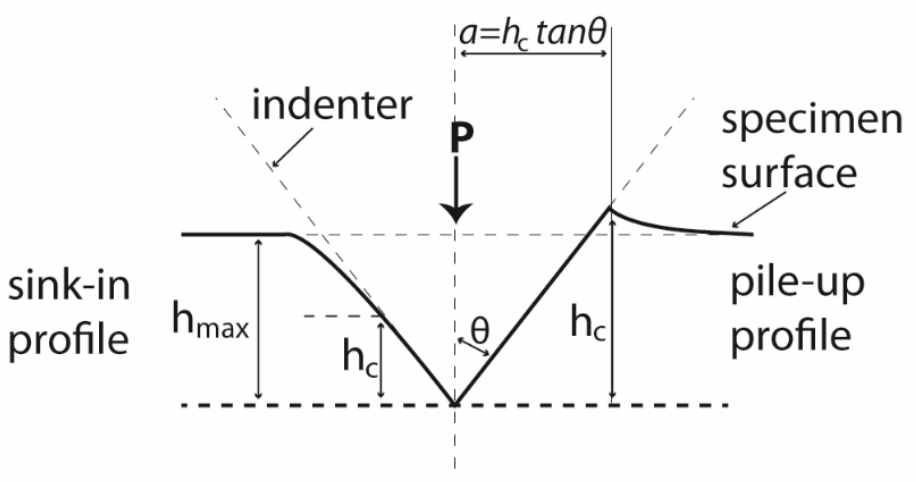

134

Table 1: Raw materials average hardness and Er values. These values are calculated taking all the measurements into account for each sample.

\begin{tabular}{|c|c|c|c|c|c|}
\hline & Spruce wood & Beech wood & Bone & Antler & Ivory \\
\hline $\begin{array}{l}\text { Reduced Modulus } \\
\qquad\left(E_{r} \text { in GPa) }\right.\end{array}$ & $1.078 \pm 0.359$ & $\begin{array}{l}27.896 \pm \\
11.062\end{array}$ & $\begin{array}{c}19.252 \pm \\
1.128\end{array}$ & $\begin{array}{c}30.562 \pm \\
5.128\end{array}$ & $\begin{array}{c}36.439 \pm \\
0.323\end{array}$ \\
\hline $\begin{array}{l}\text { Hardness } \\
\text { (H in GPa) }\end{array}$ & $0.122 \pm 0.004$ & $2.685 \pm 1.652$ & $\begin{array}{c}2.936 \pm \\
0.277\end{array}$ & $\begin{array}{c}3.253 \pm \\
0.727\end{array}$ & $\begin{array}{l}3.930 \pm \\
0.025\end{array}$ \\
\hline
\end{tabular}

Fig. 2: Material reaction during the nanoindentation process. A: Schematic drawing showing the surface displacement during indentation; B: A typical indentation $P-h$ curve where $h_{c}$ is the maximum true contact displacement during loading, $P_{\max }$ refers to the maximum load applied during the indentation cycle, $S$ is the initial unloading stiffness, $h_{f}$ is the final plastic depth from the P-h curves, $h_{s}$ is the residual depth after complete unloading and $h_{\max }$ is the indent. 


\section{Analysis}

145 An optical profilometer (S-Neox, Sensofar Metrology, Barcelona, Spain) was used to collect

146 surface topography measurements before the experimentation and then at each step of the

147 experiment. At each step, 3 to 5 measurements of different areas were taken for each sample.

148 Measurements were acquired with both a 20× objective (TU Plan Fluor EPI P; NA = 0.45; FoV

$149=872.68 \mu \mathrm{m} 655.965 \mu \mathrm{m})$ and a $50 \times$ objective $($ TU Plan Fluor EPI P; NA = 0.80; FoV $=$

$150350.88 \mu \mathrm{m} \times 264.19 \mu \mathrm{m})$. Pictures were taken using the blue LED $(530 \mathrm{~nm})$ to obtain the highest

151 resolution possible with our equipment. Only pictures with a surface measure higher than $98 \%$

152 were used for the analysis. The surface images were then analyzed using SensoMap (Standard

153 7.4). To extract surface parameters from the pictures, the filtering protocol presented in Calandra

154 et al. [37] was used: (1) extraction of the topographic layer, (2) use of a Gaussian low-pass S-

155 filter (S1 nesting index $=1.093 \mu \mathrm{m}$ for the $20 \times$ objective and $0.437 \mu \mathrm{m}$ for the $50 \times$, end effects

156 managed) to remove noise and keep the primary surface, (3) use of an F operator (polynomial of

157 degree 3) to remove the form and keep the SF surface, i.e., texture, (4) use of a Gaussian high-

158 pass L-filter ( $\mathrm{L}$ nesting index $=327.980 \mu \mathrm{m}$ for the $20 \times$ objective and $131.200 \mu \mathrm{m}$ for the $50 \times$,

159 end effects managed) to filter out the waviness and keep the SL surface, i.e., roughness, and (5)

160 setting threshold surface between 0.010 and $99.9 \%$ material ratio to remove the aberrant positive

161 and negative spikes. It is important to note, however, that sometimes that threshold was

162 readjusted depending on the persistence of the outliers. The cut-off values were calculated

163 following ISO norms [38,39] recommendations; the L nesting index used was half the size of the

164 shortest side (breadth) of the field of view, and the S1 nesting index was obtained by dividing the

165 L nesting by 300 times. Four of the ISO25178 parameters were selected to perform the statistical

166 analysis (Fig 3): arithmetic mean height $(\mathrm{Sa})$, autocorrelation length $(\mathrm{Sal})$, arithmetic mean peak 

independent, provide an overall understanding of the surface textures [40].

ISO 25178 Parameters

Arithmetic mean height $[\mathrm{Sa}]$ : Surface roughness

Autocorrelation length $[\mathrm{Sal}]$ : Length of fastest decay of autocorrelation function in any direction

Arithmetic mean peak curvature $[S p c]$ : Mean form of peaks (pointed vs rounded)

Upper material ratio $[\operatorname{Smrl}]$ : Peak material portion above core
Low
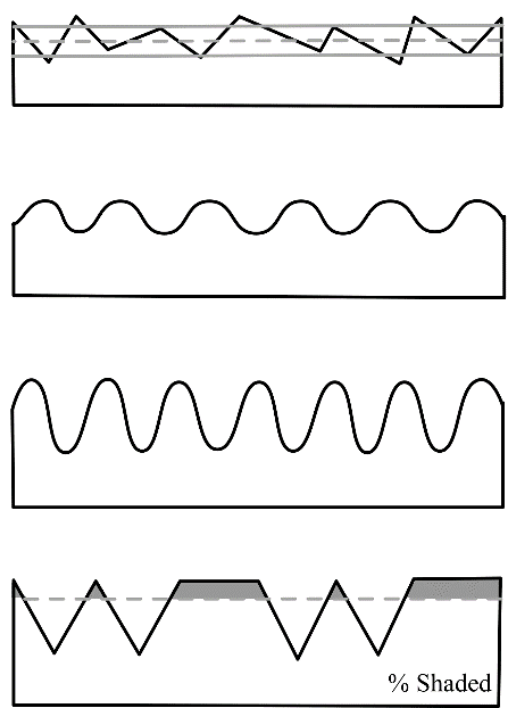

High
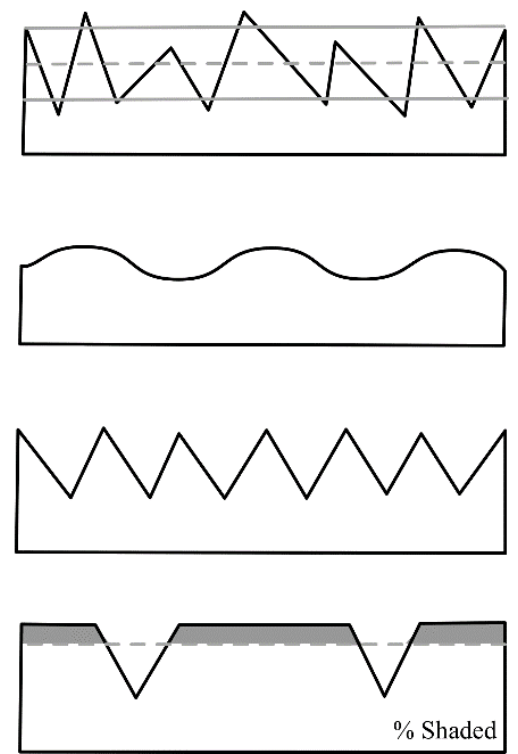

Fig. 3 Visual representation of the surface parameters. (from Martisius et al., 2018 (doi:

https://doi.org/10.1371/journal.pone.0206078.g004).

The statistical analyses were performed in the open-source software R (v. 3.5.2;[41] using the following packages: ggplot2 (v. 3.1.0; [42]), dplyr (v. 0.8.0.1; [43]), tidyr (v. 0.8.3; [44]), ggpubr (v. 0.1 .2 ; [45]), ggalt (v. 0.4.0; [46]), MASS( v. 7.3-53.1; [47]), gridExtra (v.2.3; [48]), cowplot (v. 1.1.1; [49]), rstatix(v. 0.7.0; [50]), and knitr (v. 1.31; [51]). Boxplots were produced to give a sense of the flint texture variation depending on the worked material hardness. Besides, an analysis of variance (ANOVA) and paired t-test were used to show which roughness parameters can help discriminate material types or hardness. 


\section{Direct observation results}

181 Visually, it is noticeable that polish did not develop to the same extent on all the flint bits, even

182 with the naked eye (Fig 4). According to the statistical results, the flint surface appears more

183 polished when rubbed on spruce wood and beech wood. For the other worked material, the

184 polish is sparser.

185 


\begin{tabular}{|c|c|c|}
\hline & Stone samples X30 & Microtopography of the stone sample X50 \\
\hline $\begin{array}{l}\text { Stone sample worked } \\
\text { on Spruce wood }\end{array}$ & & 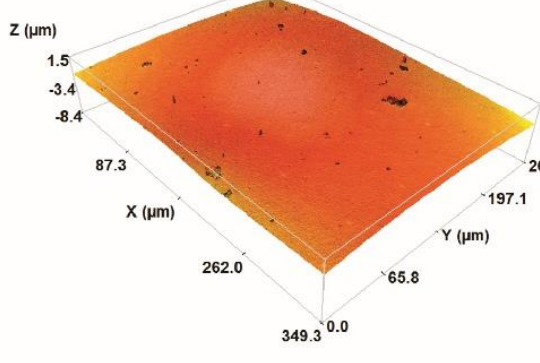 \\
\hline $\begin{array}{l}\text { Stone sample worked } \\
\text { on Beech wood }\end{array}$ & 一 & ${ }^{2(\mu \mathrm{m})}{ }^{2.9}$ \\
\hline $\begin{array}{l}\text { Stone sample worked } \\
\text { on bone }\end{array}$ & $x_{1}$ & $x$ \\
\hline $\begin{array}{l}\text { Stone sample worked } \\
\text { on antler }\end{array}$ & VI & ${ }_{5.5}^{2.8}$ \\
\hline $\begin{array}{l}\text { Stone sample worked } \\
\text { on ivory }\end{array}$ & Ping & $\begin{array}{c}\text { (um) } \\
4.3 \\
-15.4 \\
8.7 \\
\end{array}$ \\
\hline
\end{tabular}


Fig. 4 Polish development after 5 hours of use. The samples are organized by hardness order, from the softer on top of table to

188 the harder on the bottom.

189

190 Statistical results

191 An analysis of variance (ANOVA) made on each parameter show that only the $S a(p<0.001)$

192 parameter is the only one that is statistically different (Fig 5). Hence, $\mathrm{Sa}$ is the most suited of the 193 four parameters tested to understand the worked material hardness's impact on polish formation.

194 The results indicate that the worked material modifies the natural flint surface quantitatively, 195 depending on its hardness. The softer a worked material is, the more the flint surface will be 196 polished. 


\begin{tabular}{|c|c|c|c|}
\hline $\begin{array}{l}\text { tools worked on: } \\
\text { Spruce wood } \\
\text { Beech wood } \\
\text { Bone } \\
\text { Antler } \\
\text { Ivory } \\
\text { Before use }\end{array}$ & After 1 hour of work & After 3 hours of work & After 5 hours of work \\
\hline Sa parameter & 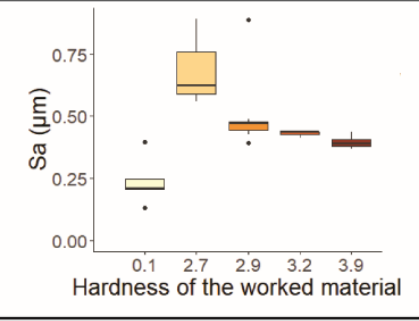 & 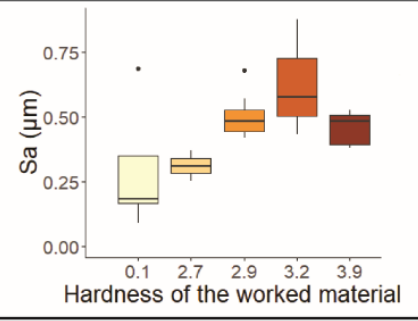 & 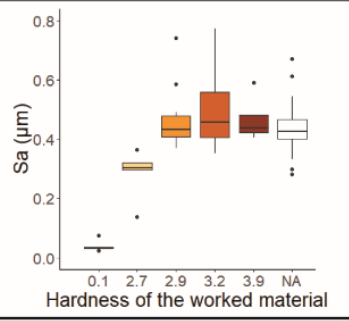 \\
\hline$\sim \sim$ & Hardness of the worked material 0. & 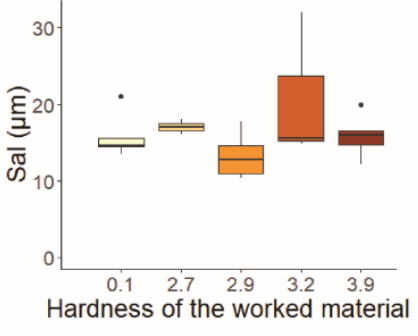 & Hardness of the worked material \\
\hline Spc parameter & 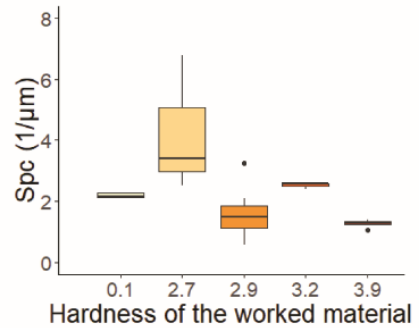 & Hardness of the worked material & 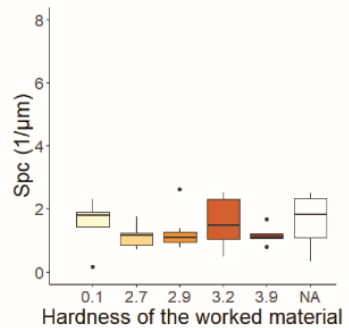 \\
\hline Smr1 parameter & 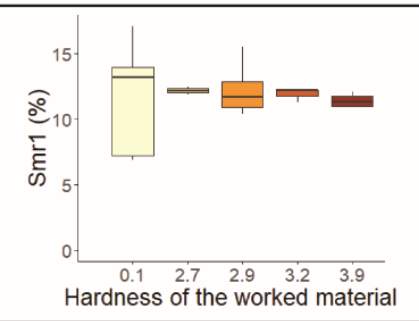 & Hardness of the worked material & $\begin{array}{c}0.1 \\
0.72 .93 .23 .9 \mathrm{NA} \\
\text { Hardness of the worked material }\end{array}$ \\
\hline
\end{tabular}

Fig. 5 Boxplots showing the evolution of flint roughness parameters Sa, Sal, Spc, and Smr1 after 1 hour, 3 hours and 5 hours of use on worked materials of various hardnesses. The diagrams in the first column represent the

200 expected microtopography for a high value (on the top part of the cell) or low value (on the bottom part of the cell)

201 for each parameter (extract from Martisius et al., 2018).

202 Paired t-tests with the Bonferroni correction results for the $S a$ parameter (see Table 2) show that

203 it is possible to differentiate a flint piece before and after use only for the softest material, the 
204 spruce wood $(p=0.001)$. It is also possible to differentiate between the two wood types $(p=$

205 0.045). However, for antler, ivory, and bone, the paired t-tests show that the means before and

206 after use were not significantly different. In term of duration of the action, the results were

207 statistically significant after 3 hours of use. However, the significance increased after 5 hours of

208 user in particular to distinguish between the two wood types.

209 Table 2: paired t-test results with the Bonferroni correction and their significance. In the adjusted p-value

210 significance column ns refers to non-significant, and the number of stars to the level of significance.

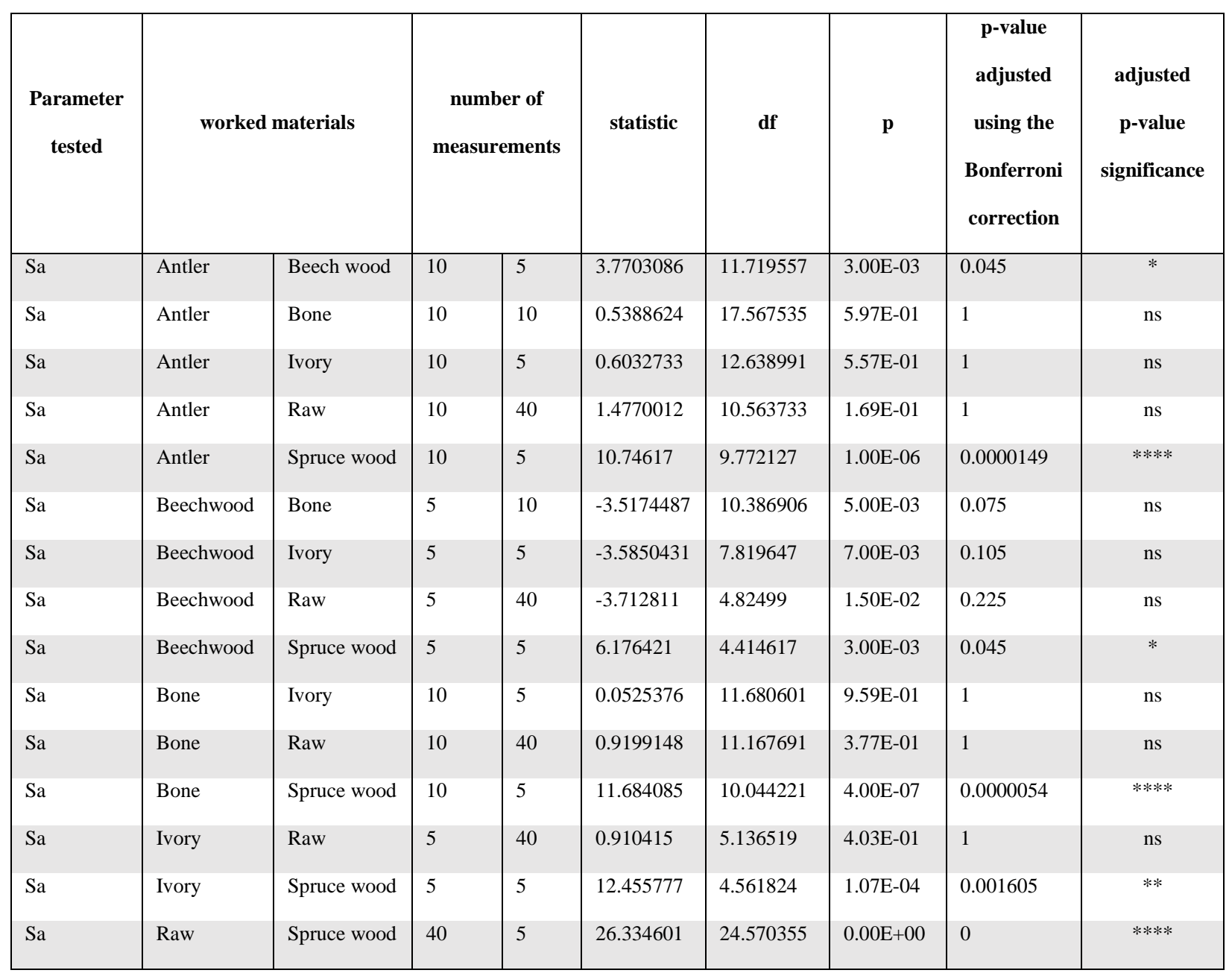

211

\section{Track analysis}


213 One reason that can explain these results could be the presence of more abrasive compounds in

214 the softer worked material such as silicate. Residual powders from the track formed by the flint

215 rubbing on the worked materials were collected and analyzed using scanning electron

216 microscopy (SEM) and X-ray analysis (EDS) (Fig 6). Silica could not be found in the EDS

217 framing the broad region of the samples, most likely because the other elements' noise was too

218 heavy to enable the detection of other compounds or because the powdered silica are too small to

219 be picked up by the EDS probe. However, we found that the interfacial powders from each raw

220 material contained flint fragments of approximately $200 \mu \mathrm{m}$.

221

222

223

224

225

226

227

228

229

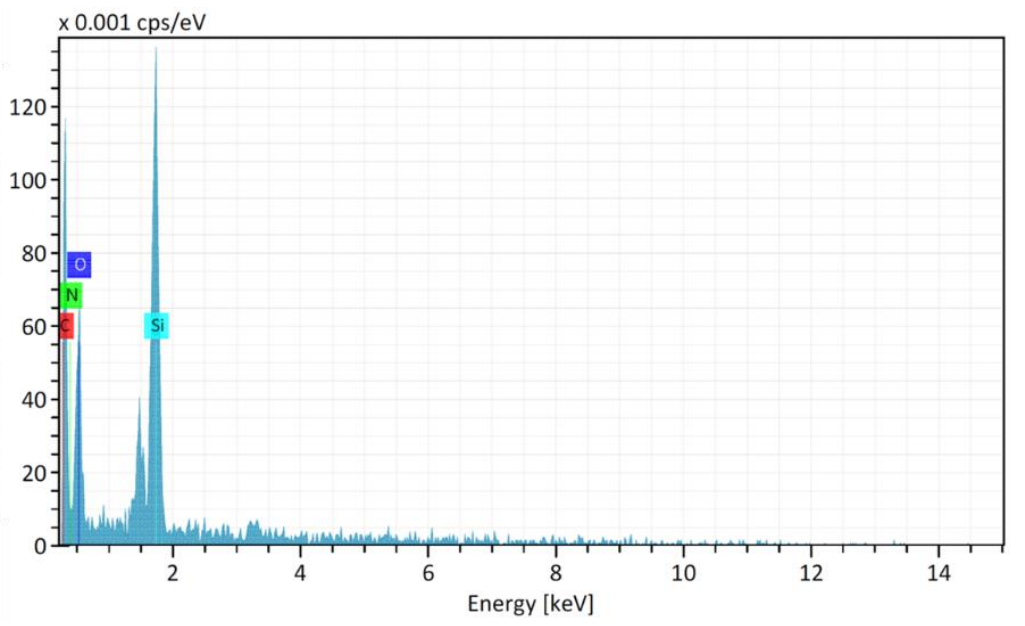

Fig. 6: X-ray microanalysis of the spruce wood powder. The red square represents the scanned area. This area is mainly composed of Silicone, suggesting that the fragment (white on the picture) is a flint fragment.

In this case, the two softer materials happen to be wood, but silica content in temperate wood species is negligible [52], whereas the apatite contained in the bone matrix are softer than the quartz in flint. At the same time, because flint was found in track powders associated with all materials, it is possible that these particles contributed most to the abrasion, since they are hard enough to scratch the stone surface. However, we do not understand exactly how and why flint particles were broken off the stone surface in the first place. Although we tried to maintain a 


\section{Discussion}

contact between two relatively flat surfaces to maximize contact area and minimize the variation in pressure distribution, because we opted for a natural raw surface, edge breakages were still possible. Moreover, it is possible that the particles we found with the aid of the EDS probe are too large to have really contributed significantly to polish development.

The basic problem in explaining what causes stone tool wear is that most worked materials (such as skins, wood, bone, etc.) are softer than stone and cannot, in theory, abrade the stone tools themselves. However, given that stone tools are, in fact, abraded by contact with softer materials [23-25], why does this happen, and which factors related to the target material play the most significant role? Our counterintuitive result suggests that the abrasion 'paradox' must be explained by either the presence of hard grits within the worked material, the presence at the interface of stone particles broken off the tool edge, or a combination of both [53]. In the second case, the ability of softer materials to cause stone particles to break off via a combination of friction heat and mechanical dislodging is likely determined by their own structure and mechanical properties. Therefore, knowing exactly to which degree these properties are responsible for creating wear is crucial for being able to recognize different worked materials.

The results obtained in this study confirm previous results stating that it is possible to differentiate between the softest material (woods) and harder ones (antler, bone, ivory) statistically. There also appears to be a statistical separation between the two types of wood, spruce and beech. However, using basic surface topography, it does not seem possible to distinguish among antler, bone, and ivory. These observations confirm previously reported 
251 difficulties in visually differentiating polish within this group, which are often reported together 252 as $\mathrm{ABI}[54,55]$.

253 An unresolved issue is that, even after 5 hours of work in our setup, the polish was barely

254 developed on harder materials, while it was almost completely flat in the wood samples. Hence,

255 for ivory, bone, and antler, it was impossible to distinguish the before and after use surfaces

256 based on changes in $S a$. In our previous work scraping beech wood [56], quantifiable differences

257 in $S a$ were only documented with higher loads, of $90 \mathrm{~N}$ and $100 \mathrm{~N}$. However, here we were able to

258 obtain changes in $S a$ for the same material, beech wood, even with the comparatively much

259 lower load of $20 \mathrm{~N}$. The discrepancy can be attributed to the difference in the ability of that

260 paper's instrument (using focus variation) to measure polish and/or, given other teams' successes

261 using focus variation [13], to the lack of a standardized surface on which to measure it. However,

262 it is also possible that higher loads or much longer working durations are required to make

263 noticeable changes to the stone surface. This could be due to processes involving dislodging

264 particles from the stone surface using friction heat, which remain to be documented

265 experimentally.

\section{Conclusion}

267 As discussed in another paper related to this experimental setup [25], polish is formed by 268 abrasion of the flint surface. Consequently, we expect that harder worked material would be 269 more effective in creating polish. However, our experiment shows that the softer the worked 270 material is (i.e., the lower the arithmetical mean height $(\mathrm{Sa})$ ), the more the polish is developed.

271 Given these results, it is imperative that other mechanical properties of materials typically

272 expected to occur in prehistoric tasks be studied. These could include the materials' own surface 
273 roughness, elasticity, fracture toughness, the presence and brittleness of embedded grits, and

274 others. In addition, longer work duration and higher loads (with different machines) can be tested

275 to obtain more polish formation on stone samples used on harder materials. Additionally, for this

276 pilot study, materials were used in the dry condition, but saturation with water and added

277 lubrication (e.g., fat) should be included in future studies.

\section{Supporting information}

279 S1 Appendix. Detailed results of the nano-indentation tests.

280 Bibliography

1. Fullagar R. Residues and usewear. In: Balme J, Paterson A, editors. Archaeology in practice: a student guide to archaeological analyses. Malden, Oxford, Carlton: Blackwell Publishing; 2006. pp. 207-234.

2. Keeley LH, Toth N. Microwear polishes on early stone tools from Koobi Fora, Kenya. Nature. 1981;293: 464-465. doi:10.1038/293464a0

3. Bamforth D. Investigating microwear polishes with blind tests: The Institute results in context. J Archaeol Sci. 1988;15: 11-23.

4. Newcomer MH, Grace R, Unger-Hamilton R. Investigating Microwear Polishes with Blind Tests. J Archaeol Sci. 1986;13: 203-217.

5. Grace R. Use-Wear Analysis: The State of the Art. Archaeometry. 1996;38: 209-229. doi:10.1111/j.1475-4754.1996.tb00771.x

6. Evans AA, Lerner H, Macdonald DA, Stemp WJ, Anderson PC. Standardization, calibration and innovation: a special issue on lithic microwear method. J Archaeol Sci. 2014;48: 1-4. doi:10.1016/j.jas.2014.03.002

7. Stemp WJ, Watson AS, Evans AA. Surface analysis of stone and bone tools. Surf Topogr Metrol Prop. 2015;4: 013001. doi:10.1088/2051-672X/4/1/013001

8. Macdonald DA, Stemp WJ, Evans AA. Exploring the microscale: Advances and novel applications of microscopy for archaeological materials. J Archaeol Sci Rep. 2018;18: 804-805.

doi:10.1016/j.jasrep.2018.02.036 
9. Ibañez JJ, González-Urquijo JE, Gibaja J. Discriminating wild vs domestic cereal harvesting micropolish through laser confocal microscopy. J Archaeol Sci. 2014;48: 96-103. doi:10.1016/j.jas.2013.10.012

10. Ibáñez JJ, Anderson PC, González-Urquijo J, Gibaja J. Cereal cultivation and domestication as shown by microtexture analysis of sickle gloss through confocal microscopy. J Archaeol Sci. 2016;73: 6281. doi:10.1016/j.jas.2016.07.011

11. Evans AA, Donahue RE. Laser scanning confocal microscopy: a potential technique for the study of lithic microwear. J Archaeol Sci. 2008;35: 2223-2230. doi:10.1016/j.jas.2008.02.006

12. Ibáñez JJ, Lazuen T, González-Urquijo J. Identifying Experimental Tool Use Through Confocal Microscopy. J Archaeol Method Theory. 2019;26: 1176-1215. doi:10.1007/s10816-018-9408-9

13. Macdonald DA. The application of focus variation microscopy for lithic use-wear quantification. J Archaeol Sci. 2014;48: 26-33. doi:10.1016/j.jas.2013.10.003

14. Pedergnana A, Calandra I, Evans AA, Bob K, Hildebrandt A, Ollé A. Polish is quantitatively different on quartzite flakes used on different worked materials. Peresani M, editor. PLOS ONE. 2020;15: e0243295. doi:10.1371/journal.pone.0243295

15. Stevens NE, Harro DR, Hicklin A. Practical quantitative lithic use-wear analysis using multiple classifiers. J Archaeol Sci. 2010;37: 2671-2678. doi:10.1016/j.jas.2010.06.004

16. Unrath G, Owen LR, van Gijn A, Moss EH, Plisson H, Vaughan P. An Evaluation of Use-Wear Studies: a Multi-Analyst Approach in Technical Aspects of Microwear Studies on Stone Tools. Early Man News. 1984; 117-176.

17. Stemp WJ, Stemp M. Documenting Stages of Polish Development on Experimental Stone Tools: Surface Characterization by Fractal Geometry Using UBM Laser Profilometry. J Archaeol Sci. 2003;30: 287-296. doi:10.1006/jasc.2002.0837

18. Evans AA. On the importance of blind testing in archaeological science: the example from lithic functional studies. J Archaeol Sci. 2014;48: 5-14. doi:10.1016/j.jas.2013.10.026

19. Pfeifer SJ, Hartramph WL, Kahlke R-D, Müller FA. Mammoth ivory was the most suitable osseous raw material for the production of Late Pleistocene big game projectile points. Sci Rep. 2019;9: 2303. doi:10.1038/s41598-019-38779-1

20. Chen P-Y, Stokes AG, McKittrick J. Comparison of the structure and mechanical properties of bovine femur bone and antler of the North American elk (Cervus elaphus canadensis). Acta Biomater. 2009;5: 693-706. doi:10.1016/j.actbio.2008.09.011

21. Currey JD, Landete-Castillejos T, Estevez J, Ceacero F, Olguin A, Garcia A, et al. The mechanical properties of red deer antler bone when used in fighting. J Exp Biol. 2009;212: 3985-3993. doi:10.1242/jeb.032292

22. Khlopachev GA, Girya EY. Sekrety drevnikh kostorezov Vostochnoy Evropy i Sibiri: priemy obrabotki bivnya mamonta i roga severnogo olenya $v$ kamennom veke [Secrets of ancient carvers of Eastern 
Europe annd Siberia: treatment ivory and reindeer antler in the Stone Age]. Saint Petersburg: St Petersburg publishing house "Nauka"; 2010.

23. Yamada S. The formation process of use-wear polishes. Traces Fonct Gestes Retrouvés. 1993;50: 2.

24. Ollé $A$, Vergès JM. SEM functional analysis and the mechanism of microwear formation. In: Longo L, Skakun N, editors. "Prehistoric Technology" 40 years later: functional studies and the Russian legacy. Oxford: Archaeopress; 2008. pp. 39-49.

25. Schmidt P, Rodriguez A, Yanamandra K, Behera RK, lovita R. The mineralogy and structure of use-

26. Astruc L, Vargiolu R, Zahouani H. Wear assessments of prehistoric instruments. Wear. 2003;255: 341-347. doi:10.1016/S0043-1648(03)00173-X

27. Lerner H, Du X, Costopoulos A, Ostoja-Starzewski M. Lithic raw material physical properties and use-wear accrual. J Archaeol Sci. 2007;34: 711-722. doi:10.1016/j.jas.2006.07.009

28. Lerner HJ. Intra-raw material variability and use-wear formation: an experimental examination of a Fossiliferous chert (SJF) and a Silicified Wood (YSW) from NW New Mexico using the Clemex Vision processing frame. J Archaeol Sci. 2014;48: 34-45. doi:10.1016/j.jas.2013.10.030

29. Yonekura K, Hotta A, Suzuki T. Influence of intrinsic properties of prehistoric stone tool materials on development of use-wear traces. Quat Res Daiyonki-Kenkyu. 2011;50: 195-204. doi:10.4116/jaqua.50.195

30. Marreiros J, Pereira T, lovita R. Controlled experiments in lithic technology and function. Archaeol Anthropol Sci. 2020;12: 110, s12520-020-01059-5. doi:10.1007/s12520-020-01059-5

31. Marreiros J, Calandra I, Gneisinger W, Paixão E, Pedergnana A, Schunk L. Rethinking Use-Wear Analysis and Experimentation as Applied to the Study of Past Hominin Tool Use. J Paleolit Archaeol. 2020;3: 475-502. doi:10.1007/s41982-020-00058-1

32. Hay J, Agee $P$, Herbert $E$. Continuous stiffness measurement during instrumented indentation testing. Exp Tech. 2010;34: 86-94. doi:10.1111/j.1747-1567.2010.00618.x

33. Li Y, An B, Fukuyama S, Yokogawa K, Yoshimura M. Surface oxidation of a $\mathrm{Nb}(100)$ single crystal by scanning tunneling microscopy. Mater Charact. 2002;48: 163-167. doi:10.1016/S10445803(02)00204-8

34. Oliver WC, Pharr GM. An improved technique for determining hardness and elastic modulus using load and displacement sensing indentation experiments. J Mater Res. 1992;7: 1564-1583. doi:10.1557/JMR.1992.1564

35. Oyen ML, Cook RF. A practical guide for analysis of nanoindentation data. J Mech Behav Biomed Mater. 2009;2: 396-407. doi:10.1016/j.jmbbm.2008.10.002 
36. Oliver WC, Pharr GM. Measurement of hardness and elastic modulus by instrumented indentation: Advances in understanding and refinements to methodology. J Mater Res. 2004;19: 3-20. doi:10.1557/jmr.2004.19.1.3

37. Calandra I, Schunk L, Rodriguez A, Gneisinger W, Pedergnana A, Paixao E, et al. Back to the edge: relative coordinate system for use-wear analysis. Archaeol Anthropol Sci. 2019 [cited 21 Feb 2019]. doi:10.1007/s12520-019-00801-y

38. Organization for Standardization. 4288-Geometrical product specifications (GPS) - surface texture: profile method-rules and procedures for the assessment of surface texture. 1996.

39. Organization for Standardization. 4287-Geometrical Product Specifications (GPS)-Surface Texture: Profile Method-Terms, Definitions and Surface Texture Parameters. International. 1997.

40. Martisius NL, Sidéra I, Grote MN, Steele TE, McPherron SP, Schulz-Kornas E. Time wears on: Assessing how bone wears using 3D surface texture analysis. PLOS ONE. 2018;13: e0206078. doi:10.1371/journal.pone.0206078

41. R Core Team. R. A language and environment for statistical computing. R Foundation for Statistical Computing, Vienna, Austria. Version 3.5.1. 2018. Available: https://www.R-project.org/

42. Wickham H. ggplot2: Elegant Graphics for Data Analysis. Springer; 2016.

43. Wickham H. Version 0.8.0.1, A Grammar of Data Manipulation. 2019. Available: https://cran.rproject.org/web/packages/R.utils/R.utils.pdf

44. Wickham H. tidyr, Version 0.8.3, Easily Tidy Data with "spread()" and "gather()" Functions. 2019. Available: https://mran.revolutionanalytics.com/web/packages/tidyr/tidyr.pdf

45. Kassambara A, Kassambara MA. Package 'ggpubr.' 2020.

46. Rudis B, Rudis MB. Package 'ggalt.' 2016.

47. Ripley B, Venables B, Bates DM, ca 1998) KH (partial port, ca 1998) AG (partial port, Firth D. MASS: Support Functions and Datasets for Venables and Ripley's MASS. 2021. Available: https://CRAN.Rproject.org/package=MASS

48. Auguie B, Antonov A. gridExtra: Miscellaneous Functions for "Grid" Graphics. 2017. Available: https://CRAN.R-project.org/package=gridExtra

49. Wilke CO. Cowplot, Streamlined Plot Theme and Plot Annotations for "ggplot2", Version 0.9.4. 2019. Available: https://cran.r-project.org/web/packages/cowplot/cowplot.pdf

50. Kassambara A. rstatix: Pipe-Friendly Framework for Basic Statistical Tests. 2021. Available: https://CRAN.R-project.org/package=rstatix

51. Xie [aut $Y$, cre, Vogt $A$, Andrew A, Zvoleff A, http://www.andre-simon.de) AS (the $C$ files under inst/themes/ were derived from the $H$ package, et al. knitr: A General-Purpose Package for Dynamic Report Generation in R. 2021. Available: https://CRAN.R-project.org/package=knitr 
52. Fullagar RLK. The role of silica in polish formation. J Archaeol Sci. 1991;18: 1-24. doi:10.1016/0305-4403(91)90076-2

53. Stachowiak GW, Batchelor AW. Engineering tribology. Fourth edition. Oxford: Elsevier/Butterworth-Heinemann; 2014.

54. Sano K. Functional variability in the Magdalenian of north-western Europe: A lithic microwear analysis of the Gönnersdorf K-II assemblage. Quat Int. 2012;272-273: 264-274. doi:10.1016/j.quaint.2012.02.057

55. Iwase A. A functional analysis of the LGM microblade assemblage in Hokkaido, northern Japan: A case study of Kashiwadai 1. Quat Int. 2016;425: 140-157. doi:10.1016/j.quaint.2016.04.008

56. Pfleging J, lovita R, Buchli J. Influence of force and duration on stone tool wear: results from experiments with a force-controlled robot. Archaeol Anthropol Sci. 2018 [cited 9 Apr 2019]. doi:10.1007/s12520-018-0729-0 